\title{
Cost-effectiveness of pembrolizumab versus chemotherapy as first-line treatment in PD-L1-positive advanced non-small-cell lung cancer in the USA
}

\author{
Min Huang*,1, Gilberto de Lima Lopes², Ralph P Insinga1', Thomas Burke ${ }^{1}$, Flavia \\ Ejzykowicz ${ }^{1}$, Ying Zhang ${ }^{3}$ \& Josephine L Feliciano ${ }^{4}$ \\ ${ }^{1}$ Center for Observational \& Real World Evidence (CORE), Merck \& Co., Inc., Kenilworth, NJ 07033, USA \\ ${ }^{2}$ Sylvester Comprehensive Cancer Center, University of Miami \& the Miller School of Medicine, Miami, FL 33136, USA \\ ${ }^{3}$ HTA Statistics Europe, Merck Sharp \& Dohme, 1200 Brussels, Belgium \\ ${ }^{4}$ The Sidney Kimmel Comprehensive Cancer Center, Johns Hopkins University, Baltimore, MD 21287, USA \\ *Author for correspondence: min_huang@merck.com
}

\begin{abstract}
Aim: This analysis aimed to evaluate the cost-effectiveness of pembrolizumab monotherapy as first-line treatment in advanced non-small-cell lung cancer patients with a programmed death ligand 1 (PD-L1) tumor proportion score $\geq 1 \%$ from a US payer perspective. Materials \& methods: A partitioned survival model was developed using efficacy and safety data from the KEYNOTE-042 trial and projected over 20 years. Costs accounted for treatment, toxicity and disease management. Quality-adjusted life-years (QALYs) and incremental cost-effectiveness ratios were reported. Results: Pembrolizumab resulted in an expected gain of 0.60 life years and 0.49 QALYs compared with platinum-based chemotherapy. The incremental costeffectiveness ratio was US $\$ 130,155 /$ QALY. Conclusion: Pembrolizumab is projected to be cost-effective compared with platinum-based chemotherapy as first-line treatment for advanced non-small-cell lung cancer with PD-L1 tumor proportion score $\geq 1 \%$.
\end{abstract}

First draft submitted: 3 October 2019; Accepted for publication: 31 October 2019; Published online: 18 November 2019

Keywords: advanced NSCLC • cost-effectiveness • PD-L1-positive • pembrolizumab

Lung cancer is the leading cause of cancer deaths in USA and worldwide [1]. Non-small-cell lung cancer (NSCLC), which represents $85-90 \%$ of lung cancer, is usually diagnosed with advanced disease and half the patients initially treated for early-stage NSCLC subsequently relapse, which often leads to poor long-term prognosis [2]. Until recently, platinum-based chemotherapy was the standard of care for the first-line treatment of advanced or metastatic NSCLC [3].

The emergence of immunotherapy has expanded treatment options for NSCLC patients [4]. Pembrolizumab is a humanised IgG4 monoclonal antibody programmed death protein-1 (PD-1), and has demonstrated significant clinical benefit for the treatment of advanced or metastatic NSCLC in several randomized clinical trials [5-9]. In October 2016, pembrolizumab monotherapy received approval by the US FDA for the treatment of metastatic NSCLC whose tumors express PD-1 ligand (PD-L1) (tumor proportion score $[\mathrm{TPS}] \geq 1 \%$ ) in patients with disease progression on or after platinum-containing chemotherapy based on KEYNOTE-010, and for metastatic NSCLC patients whose tumors have high PD-L1 expression (TPS $\geq 50 \%$ ) and who have not had prior systemic chemotherapy treatment for metastatic NSCLC based on KEYNOTE-024. Pembrolizumab was subsequently approved in USA in 2018 in combination with platinum-based chemotherapy for first-line treatment of metastatic NSCLC, regardless of PD-L1 expression status based on KEYNOTE 189 (nonsquamous) and 407 (squamous) [10]. In April 2019, the FDA expanded the pembrolizumab monotherapy indication for first-line treatment of patients with locally advanced or metastatic NSCLC with a PD-L1 TPS $\geq 1 \%$ and no epidermal growth factor receptor or anaplastic lymphoma kinase genomic tumor aberrations, based on data from KEYNOTE-042 [11,12]. 
Table 1. Distribution of platinum-based chemotherapy regimens in KEYNOTE-042.

\begin{tabular}{|c|c|c|c|c|c|}
\hline Regimen & Therapies & Dose & Frequency & Duration & Distribution \\
\hline \multirow[t]{2}{*}{$1^{\dagger}$} & Carboplatin & AUC 5-6 & $\begin{array}{l}\text { Day } 1 \text { of every 3-week } \\
\text { cycle }\end{array}$ & Maximum six cycles & $51 \%$ \\
\hline & Pemetrexed & $500 \mathrm{mg} / \mathrm{m}^{2}$ & $\begin{array}{l}\text { Day } 1 \text { of every } 3 \text {-week } \\
\text { cycle }\end{array}$ & Maximum six cycles $\ddagger$ & \\
\hline \multirow[t]{2}{*}{2} & Carboplatin & $A \cup C 5-6$ & $\begin{array}{l}\text { Day } 1 \text { of every } 3 \text {-week } \\
\text { cycle }\end{array}$ & Maximum six cycles & $49 \%$ \\
\hline & Paclitaxel & $200 \mathrm{mg} / \mathrm{m}^{2}$ & $\begin{array}{l}\text { Day } 1 \text { of every } 3 \text {-week } \\
\text { cycle }\end{array}$ & Maximum six cycles $\S$ & \\
\hline \multicolumn{6}{|c|}{$\begin{array}{l}\dagger \text { Permitted for patients with nonsquamous histology only. } \\
\text { ¥Followed by optional pemetrexed maintenance therapy given at a dose of } 500 \mathrm{mg} / \mathrm{m}^{2} \text { every } 3 \text { weeks. } \\
\text { § Followed by optional pemetrexed maintenance therapy given at a dose of } 500 \mathrm{mg} / \mathrm{m}^{2} \text { every } 3 \text { weeks (patients with nonsquamous histology only). } \\
\text { AUC: Area under the curve. }\end{array}$} \\
\hline
\end{tabular}

The KEYNOTE-042 trial is a multicenter, international, randomized, open-label, controlled trial of pembrolizumab monotherapy versus platinum-based chemotherapy in previously untreated advanced NSCLC patients whose tumors express PD-L1 (TPS $\geq 1 \%$ ). The trial demonstrated that advanced NSCLC patients with PD-L1 TPS $\geq 1 \%$ treated with pembrolizumab $200 \mathrm{mg}$ every 3 weeks had superior overall survival (OS) as compared with those treated with platinum-based chemotherapy, with a hazard ratio for death of 0.81 ( $95 \% \mathrm{CI}$ : 0.71-0.93; $\mathrm{p}=0.0018$ ) [11]. Grade 3-5 treatment-related adverse events (AEs) were less common with pembrolizumab than chemotherapy. The data supported extending KEYTRUDA monotherapy to a wider population with previously untreated NSCLC.

This study used KEYNOTE-042 data to evaluate the cost-effectiveness of pembrolizumab monotherapy compared with platinum-based chemotherapy in an advanced NSCLC patient population with TPS $\geq 1 \%$. The analysis was designed to assist value-based reimbursement decisions for US third-party public healthcare payers.

\section{Materials \& methods}

The methods employed in this analysis was consistent with those in previously published health economic evaluations of pembrolizumab [13]. A partitioned-survival model [14] was developed to evaluate the economic implications of treating PD-L1-positive (TPS $\geq 1 \%$ ) advanced NSCLC patients with pembrolizumab compared with platinumbased chemotherapy. The main outcome measure was the incremental cost-effectiveness ratio (ICER), expressed as a cost per quality-adjusted life-year (QALY) gained; the incremental cost per life-year (LY) gained was also reported.

Efficacy, safety and utility data of the model were derived from the KEYNOTE-042 trial [12], with a cut-off date of 4 September 2018 and median follow-up of 14.0 months (range: 0.1-43.7 months).

\section{Population \& interventions}

The target population in the model was based on the KEYNOTE-042 trial population. Patients were at least 18 years of age (average age of 64 years); had a histologically or cytologically confirmed diagnosis of advanced or metastatic NSCLC, without an epidermal growth factor receptor sensitizing mutation or an anaplastic lymphoma kinase translocation; had a PD-L1 positive (TPS $\geq 1 \%$ ) tumor as determined by the PD-L1 IHC 22C3 pharmDx assay (Agilent Technologies, CA, USA at two central laboratories (one for China; one for rest of world); and received no prior systemic chemotherapy treatment for their advanced/metastatic NSCLC. Randomization of the trial was stratified by PD-L1 expression status (TPS $\geq 50 \%$ vs TPS: $1-49 \%$ ), by region of enrolment (east Asia vs rest of world), ECOG performance status score (0 vs 1) and histology (squamous vs nonsquamous).

Patients in KEYNOTE-042 were randomized to pembrolizumab $200 \mathrm{mg}$ once every 3 weeks for up to 35 cycles, or the investigator's choice of one of two platinum-based chemotherapies for a maximum of six cycles. Nonsquamous patients in the chemotherapy arm without progressive disease after completion of chemotherapy were eligible to receive pemetrexed maintenance. The chemotherapy regimens included pemetrexed + carboplatin, and paclitaxel + carboplatin (details in Table 1$)$.

\section{Model structure}

The partitioned-survival model was constructed with three mutually exclusive health states, progression-free (starting state), progressive disease and death, as shown in the transition diagram in Figure 1. Note that within a partitioned 
Figure 1. Model states and transitions. AE: Adverse event.

Reproduced from [13] Creative Commons Attribution-NonCommercial 4.0International License (http://creativecommons.org/licenses/by-nc/4.0/).

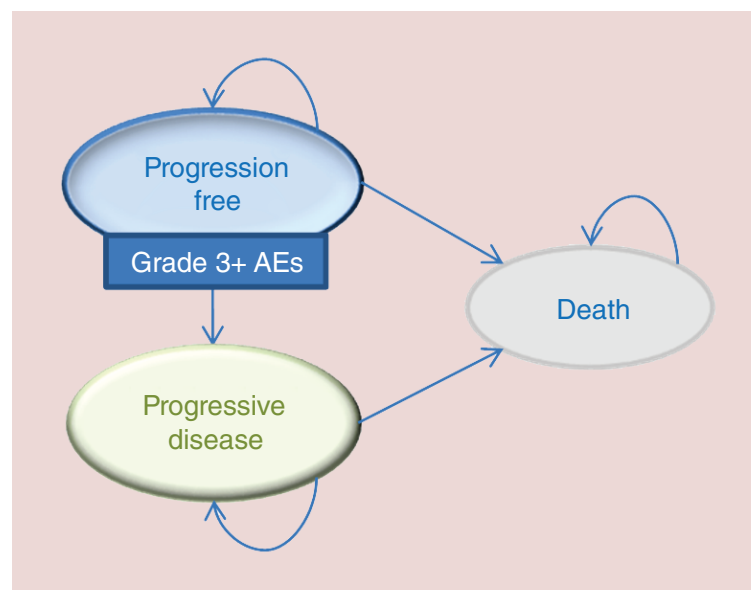

survival framework, individual transition probabilities between the progression-free/progressed disease states and death are not specifically quantified. The proportion of patients in each health state over time was estimated using the progression-free survival (PFS) and OS survival data directly. The time in each health state was used to estimate cumulative total costs and health outcomes over the time horizon for a cohort of patients receiving each intervention. Outcomes and costs of AEs with toxicity grade 3-5, based on National Cancer Institute Common Terminology Criteria for AEs version 4.0 [15], were incorporated in the model. Based on this guideline, an AE is classified as grade 3 if it is severe or medically significant but not immediately life-threatening; hospitalization or prolongation of hospitalization indicated; disabling; limiting selfcare activities of daily living. A grade $4 \mathrm{AE}$ is defined as having life-threatening consequences, or urgent intervention indicated. Death related to AE is classified as grade 5 [15].

\section{Time horizon, discount rate \& perspective}

To ensure important differences in costs and outcomes between the interventions were considered, a time horizon long enough to accommodate patients' life expectancy (a 20-year time horizon for the base case) was selected. A scenario analyses explored the effect of shorter time horizons ( 5 and 10 years), in view of the short life expectancy of patients with advanced NSCLC.

Costs and outcomes were discounted at 3\% per year as recommended by the US Guidelines for the Economic Evaluation of Health Technologies [16]. Discount rates of 0 and 5\% were explored in scenario analyses.

The analysis was conducted from a US third-party public healthcare payer perspective.

\section{Clinical parameters}

The model effectiveness parameters were derived based on the patient-level data for time-on-treatment (ToT), PFS and OS from KEYNOTE-042.

In KEYNOTE-042, disease progression was assessed by masked independent central review according to Response Evaluation Criteria in Solid Tumors, version 1.1 [17]. Patients with radiographic disease progression who were clinically stable could continue study treatment until progression was confirmed on a scan obtained at least 4 weeks later.

ToT data from the KEYNOTE-042 trial were analysed to determine treatment duration and cost for both pembrolizumab and chemotherapy arm. Patients deriving clinical benefit could continue pembrolizumab beyond progression or discontinue treatment due to a variety of factors. In the chemotherapy arm, a large proportion $(253 / 615)$ of patients discontinued treatment before disease progression after they received a maximum of six cycles of platinum-based chemotherapy or experienced unacceptable AEs. Almost all patients ( $94 \%$ in pembrolizumab and $97 \%$ in chemotherapy arm) discontinued study drug within the trial follow-up duration (including those who completed the treatment course), thus avoiding the need for extrapolation.

Parametric models were fitted to patient-level data of PFS and OS, to extrapolate from the trial period to the 20-year model time horizon. The survival curve fitting was carried out in line with the published guideline by NICE Decision Support Unit [18]. The standard parametric models were fitted, including the Weibull, the exponential, the lognormal, the log logistic, the generalized gamma and the Gompertz distributions. The best- 


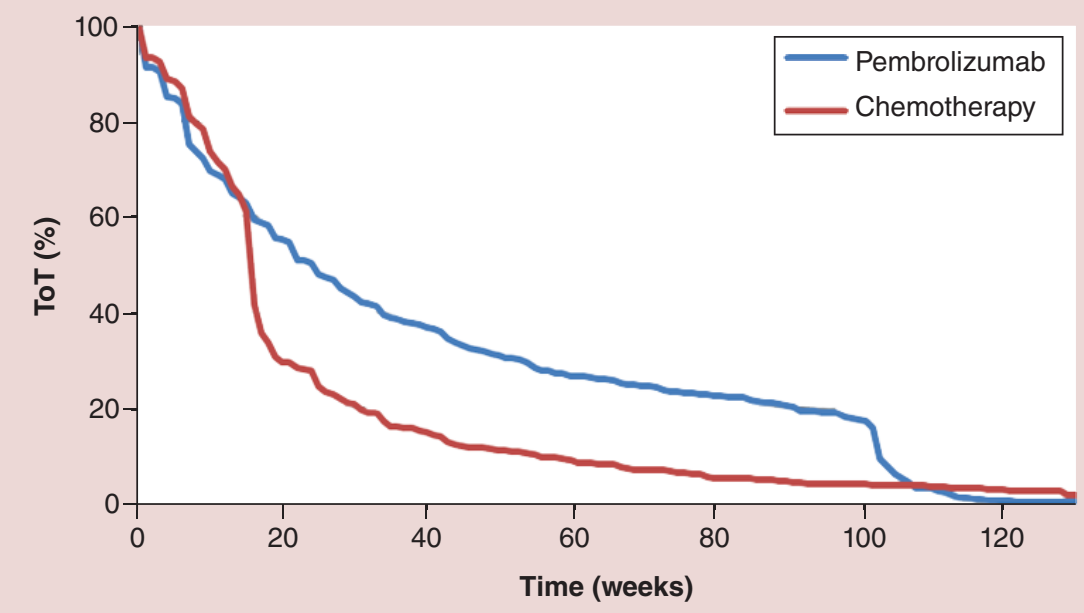

Figure 2. Modeled time-on-treatment from KEYNOTE-042 for pembrolizumab and platinum-based chemotherapy arms.

ToT: Time-on-treatment.

fitting parametric distributions was selected using statistical tests based on the Akaike information criterion and the Bayesian information criterion, combined with visual inspection The clinical plausibility of the extrapolated results was considered in selecting the final parametric distributions for the model. Scenario analyses were conducted to test the robustness of the model results to alternative parametric model fittings.

\section{Time-on-treatment}

The Kaplan-Meier (KM) data from KEYNOTE-042 were used directly in the model to inform ToT, as shown in Figure 2. Patients treated with pembrolizumab were assumed to be treated for a maximum of 35 cycles or 2 years as per trial protocol and FDA-approved label [10].

\section{Progression-free survival}

The PFS KM probabilities from KEYNOTE-042 were used directly up to Week 9 and parametric functions were fitted thereafter. This was because the first radiologic tumor response assessment was conducted at Week 9, which resulted in a protocol-driven drop in PFS curves at this point and made the fitting of a one-piece parametric curve challenging. The Weibull distribution provided the best fit for pembrolizumab, and the best-fit for chemotherapy was exponential (Figure 3).

\section{Overall survival}

Within-trial crossover was not permitted in KEYNOTE-042, however, 132/637 (20.7\%) patients in the chemotherapy arm received pembrolizumab or other anti-PD1/PD-L1 therapy after discontinuation of study drug. A simplified two-stage approach as described by Latimer $e t$ al. $[19,20]$ was implemented to perform an OS analysis adjusting for switching in the chemotherapy arm. Through this approach, the OS treatment effect estimate was adjusted to remove the impact of treatment switching to anti-PD1/PD-L1 therapies.

Two datasets are available for use in the OS analysis, the intention-to-treat (ITT) dataset included patients that switched from chemotherapy to pembrolizumab following disease progression, and a 'switching-adjusted' dataset that used the two-stage adjustment method to adjust the survival benefit of patients that switched to pembrolizumab.

The ITT dataset was used in the base-case analysis, as it better represents the real-world situation given the existing FDA approval of pembrolizumab and other PD1/PD-L1 inhibitors as second-line therapies for metastatic NSCLC expressing PD-L1 [10]. The OS impact of subsequent therapies was assumed to be reflected within the OS KM data from KEYNOTE-042. The associated treatment costs of the subsequent PD1/PD-L1 inhibitors were 


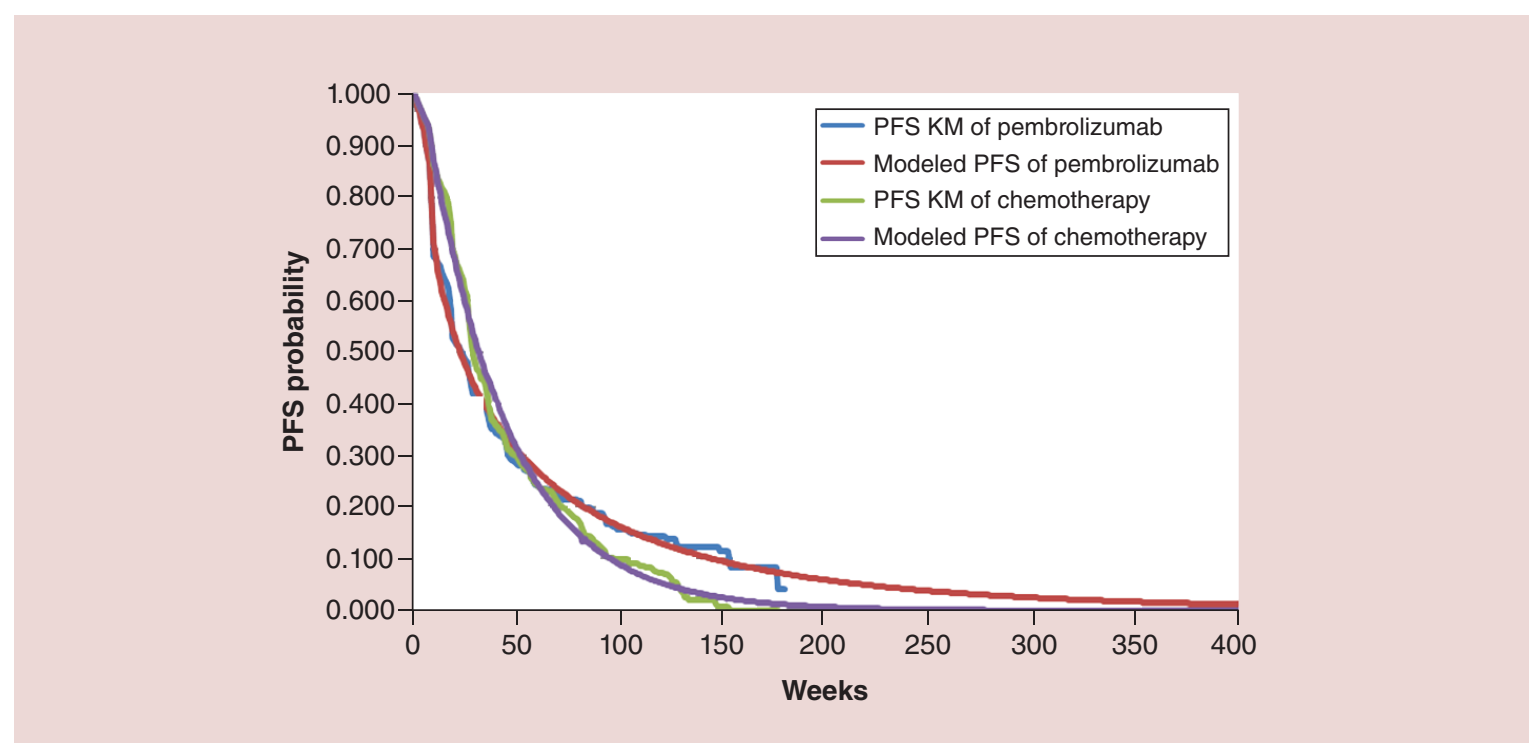

Figure 3. Modeled progression-free survival from KEYNOTE-042 for pembrolizumab and platinum-based chemotherapy arms.

KM: Kaplan-Meier; PFS: Progression-free survival.

also incorporated. The switching-adjusted dataset was explored in a scenario analysis to evaluate pembrolizumab compared with chemotherapy without subsequent use of PD1/PD-L1 inhibitors.

For the OS outcomes, the standard parametric curves tested did not provide a good visual fit to the observed KM data, so a two-phase piecewise model was applied. For both treatment arms, inspection of the cumulative hazard plots showed the changes in hazard to not be constant over time. Results suggested a piecewise model with a turning point at 33 weeks would be appropriate. Week 33 is also the point where KM curves of pembrolizumab and chemotherapy cross, with higher OS in the pembrolizumab arm thereafter. A number of cut-off points were explored in the scenario analysis. Cut-off points week 28 and 42 suggested by estimating structural changes to the KM curve using Chow tests [21] were assessed. Additional cut-off point week 52 was also tested to evaluate the impact of the uncertainty of cut-off points. These cut-offs lead to consistent results (as shown in section 3.3). Cut-off points beyond week 52 were not recommended considering the rate of censoring after that time point.

The area under the KM curve was used directly for the first phase until the cut-off, and an exponential distribution was used to estimate OS for up to 4 years of follow-up. A constant hazard rate was derived from long-term external data for patients diagnosed with stage IIIB, and for those with stage IV NSCLC from the Surveillance, Epidemiology and End Results (SEER) database. The proportion of stage IIIB versus stage IV patients in the KEYNOTE-042 trial was applied to derive the (weighted) average hazard rate. We then applied the average hazard rate to the OS models in both the pembrolizumab and chemotherapy arms after year 4 [22]. The modeled OS curves are shown in Figure 4.

\section{Subsequent therapies}

The cost of subsequent therapies was incorporated in the model. There were $44 \%$ of patients in the pembrolizumab arm and $49 \%$ for chemotherapy that received subsequent therapy after treatment discontinuation in KEYNOTE042. The distribution and duration of subsequent therapies was based on data from KEYNOTE-042. Any PD$1 / \mathrm{PD}-\mathrm{L} 1$ inhibitors, as well as the seven most commonly used 2L chemotherapy regimens (which were used by $\geq 2 \%$ patients in either arm) and the most commonly used $3 \mathrm{~L}$ and $4 \mathrm{~L}+$ chemotherapy regimen were included in the model. The percentages of patients receiving any other treatment were redistributed among the top therapies to ensure that the total proportion receiving subsequent therapy in either arm was aligned with the trial data. In the scenarios with switching adjustment, all such patients were assumed to receive chemotherapy. 
(A)

Modeled OS of pembrolizumab and chemotherapy (ITT)

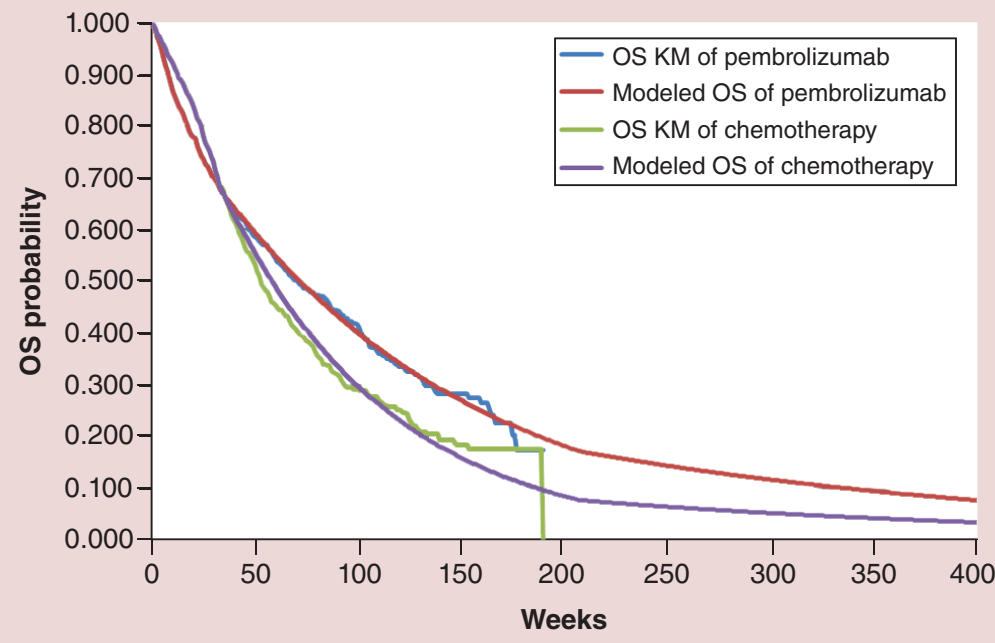

(B) Modeled OS of pembrolizumab and chemotherapy (switching adjusted)

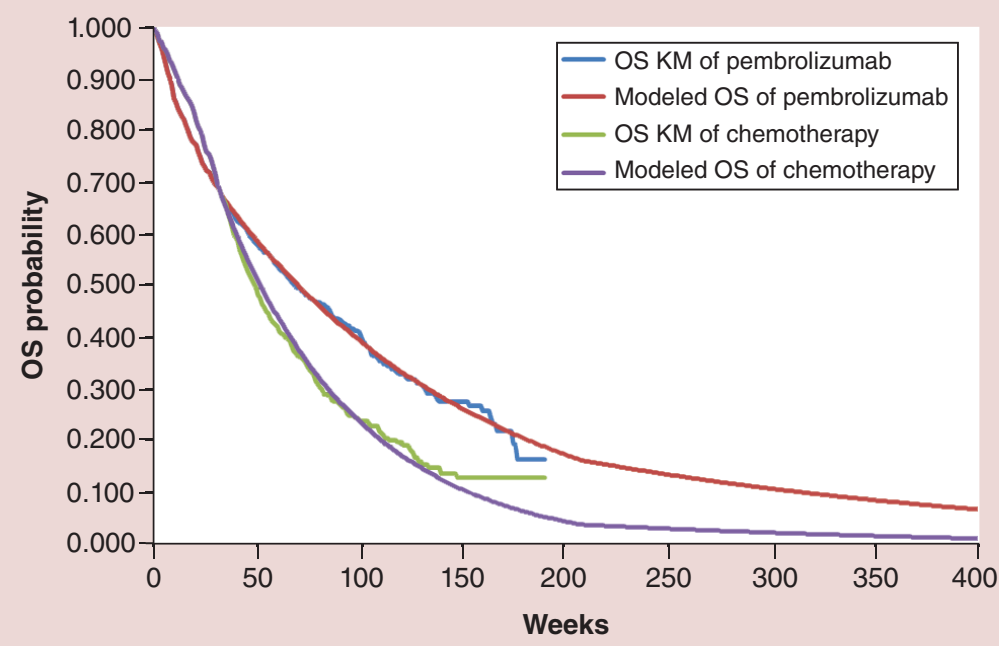

Figure 4. Modeled overall survival with the hazard rate from Surveillance, Epidemiology and End Results data applied after year 4. (A) An intention-to-treat analysis without switching adjustment. (B) Switching adjusted. KM: Kaplan-Meier; OS: Overall survival; SEER: Surveillance, epidemiology and end results program.

\section{Adverse events}

The model included all-cause AEs of Grade 3-5 reported in $\geq 5 \%$ of patients in KEYNOTE-042 for either arm. Pneumonitis, an immune-mediated AE with a high management cost, was included per clinicians' input. Model input data for each $\mathrm{AE}$ are shown in Table 2.

\section{Utility data}

Health utilities were not collected in the KEYNOTE-042 trial. The utility data used in the model (Table 2) were based on EQ-5D-3L data collected in the KEYNOTE-024 trial (with data cut-off date of 10 July 2017), which is a randomized Phase III trial comparing pembrolizumab to platinum-based chemotherapy in patients with previously untreated metastatic NSCLC with PD-L1 positive (TPS $\geq 50 \%$ ) tumors. This was considered as an appropriate data source as the two trials had similar inclusion criteria, and previous research has suggested that utilities for specific health states do not vary substantially by PD-L1 status [25].

The time-to-death approach, reflecting the decline in cancer patients' quality of life as they approach death, was utilized to model health state utilities in the model as originally described by Hatswell et al. [26]. Four time-to-death 
Table 2. Key input data.

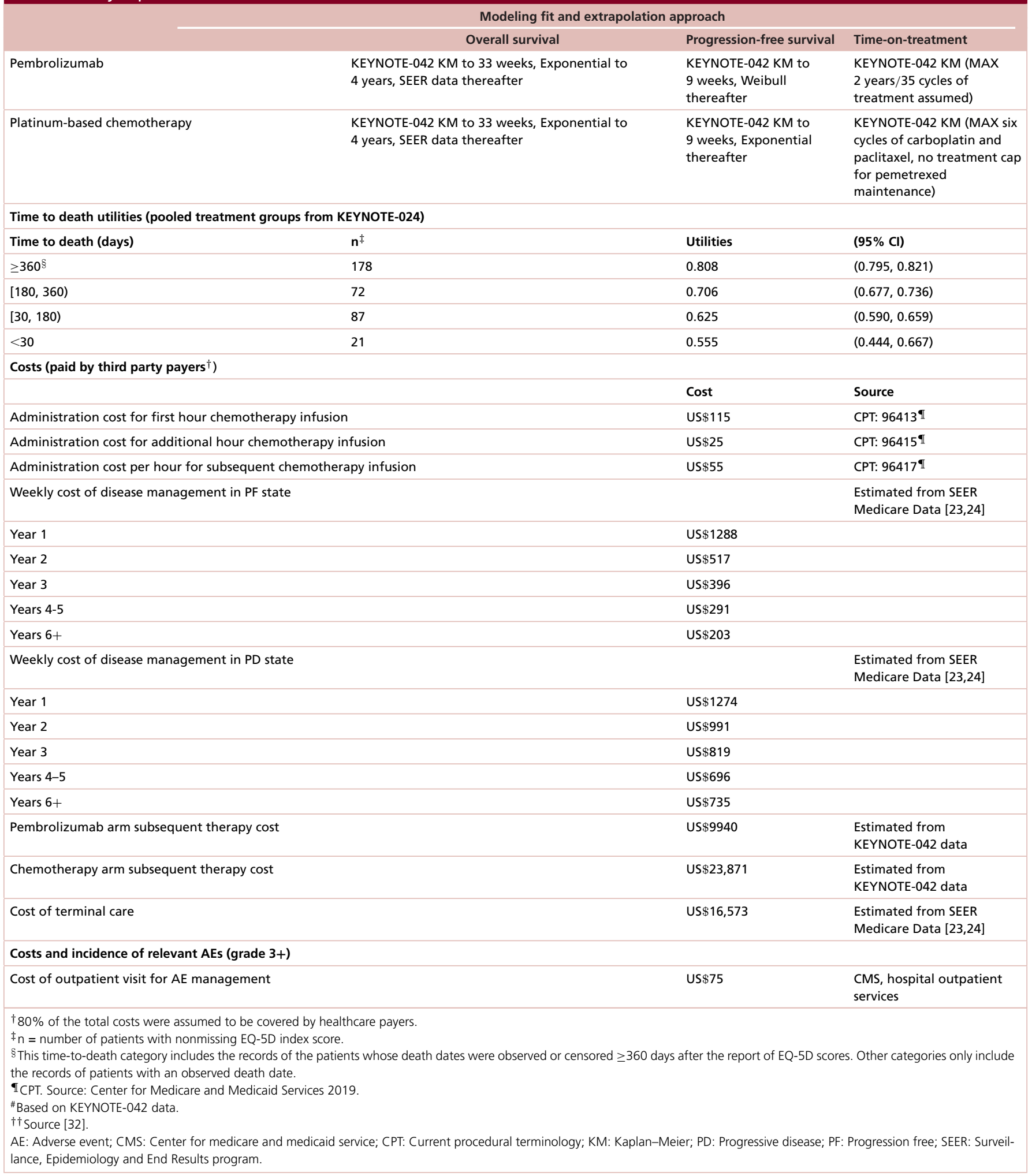


Table 2. Key input data (cont.).

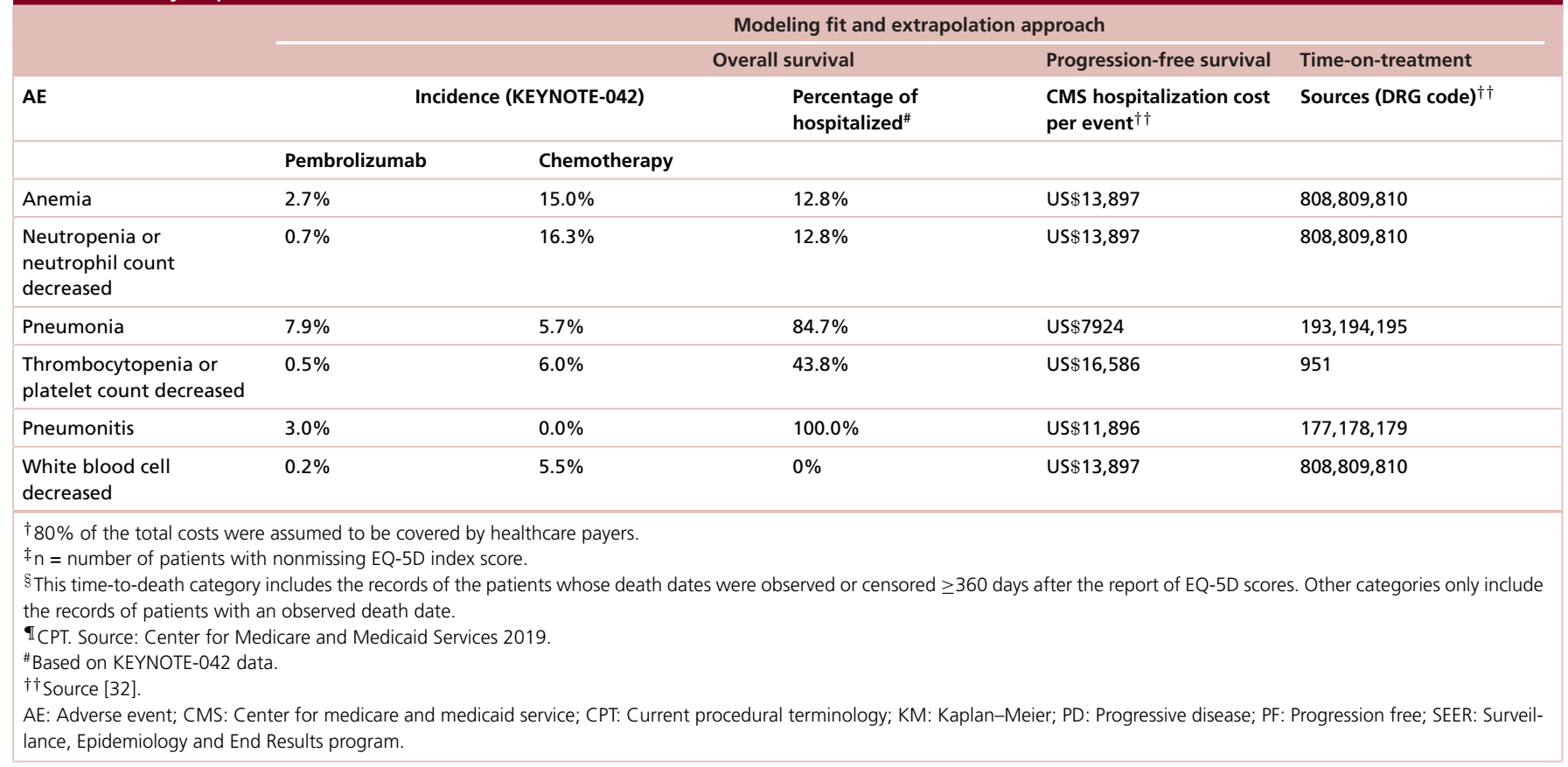

categories ( $\geq 360,180-359,30-179$ days and under 30 days) were examined for use in the model base case. Utility values from the pooled treatment groups were used as there were no significant differences in each time-to-death category between the treatment arms [13].

\section{Resource utilization \& cost inputs}

The cost inputs considered in the cost-effectiveness analysis were regimen related costs including drug acquisition, administration and premedication costs, disease management costs, subsequent therapy costs, terminal care costs and AE management costs (summarized in Table 2). It was assumed that $80 \%$ of healthcare costs would be paid by healthcare payers [27]. All costs were updated to 2019 US\$ using the Medical Care Consumer Price Index [28].

\section{Regimen related costs}

Pembrolizumab is available in single-use vials of $100 \mathrm{mg}$ and is administered at a dose of $200 \mathrm{mg}$ every 3 weeks. The list price for pembrolizumab at the time of analysis was US $\$ 4650$ per $100-\mathrm{mg}$ vial, therefore the cost per dose was US $\$ 9300$ [29]. Dosage of chemotherapy regimens was based on patients' body surface area. The average number of vials per dose of each regimen was calculated using the body surface area distribution (mean: $1.84 \mathrm{~m}^{2}$; SD: $0.26 \mathrm{~m}^{2}$ ) of metastatic NSCLC patients in the Flatiron database [30]. The cost of platinum-based chemotherapy was US $\$ 3413$ per dose, based on the distribution of each regimen in KEYNOTE-042. The cost per dose of pemetrexed maintenance was estimated at US $\$ 6562$. Costs of anti-emetic prophylaxis were identified from National Comprehensive Cancer Network (NCCN) guidelines and estimated at US $\$ 346$ and US $\$ 11$ per cycle for carboplatin and paclitaxel, respectively [31]. A total of $80 \%$ of the drug cost was assumed to be paid by healthcare payers. Drug administration costs for intravenous therapy (IV) infusions based on 2019 Center for Medicare and Medicaid Services payment rates [25] are shown in Table 2.

The number of treatment cycles in the model was based on the estimated ToT. Treatment duration was modeled per KEYNOTE-042, and consistent with the FDA-approved label for up to six cycles for platinum chemotherapy and up to 2 years (or 35 cycles) for pembrolizumab [10]. Patients sometimes miss or delay a dose, which results in their actual treatment having fewer cycles than it should. To adjust for this, data from KEYNOTE-042 were used to determine the percentage of actual treatment cycles received versus expected. The total regimen related cost was adjusted by this percentage ( $98.3 \%$ for pembrolizumab and $98.2 \%$ for chemotherapy). 


\section{Disease management costs \& terminal care costs}

The average weekly costs of disease management in the progression-free as well as progressive disease states were estimated based on 2013 SEER Medicare data for metastatic NSCLC patients [23,24]. Cost elements included inpatient, emergency care, outpatient, skilled nursing facility and hospice visits. Costs from the last 30 days of life and cost of anticancer therapy were excluded. Cost inputs used in the model were stratified by years 1, 2, 3, 4-5 and $6+$ following first-line treatment initiation.

The one-time cost of terminal care, assumed to include health care in the last 30 days of life, was also derived from 2013 SEER Medicare data. This cost included hospice, hospitalization, palliative chemotherapy, physician consultations and laboratory and diagnostic tests. The resultant cost updated to 2019 USUS\$ was US\$16,573.

\section{Subsequent therapy costs}

Using the prevalence of subsequent therapy use, and number of doses estimated from average treatment duration for each subsequent therapy received in KEYNOTE-042, the average cost of post-progression active therapy was estimated at US $\$ 9940$ per patient in the pembrolizumab arm and US $\$ 23,871$ per patient in the chemotherapy arm, including administration costs. The cost was incorporated in the model as a one-off cost upon treatment discontinuation.

\section{AE management costs}

Incidence and costs of selected grade 3+ AEs are summarized in Table 2. The rates of hospitalization associated with each individual AE were obtained from the trial. The hospitalization costs for managing the AE events were from 2019 Center for Medicare and Medicaid Services payment rates [32]. For the remaining nonhospitalized patients, the cost of an outpatient physician visit was assigned [33]. Based on incidences of AEs and management costs, the total average cost per patient for managing AEs was estimated to be US $\$ 987$ for pembrolizumab and US $\$ 1403$ for chemotherapy.

\section{Sensitivity analyses}

One-way deterministic sensitivity analyses and probabilistic sensitivity analyses (PSA) were conducted to evaluate the sensitivity of the model to uncertainty in model parameters (Table 3). Multiple scenario-based sensitivity analyses were also performed to explore the impact of key model assumptions including treatment switching, PDL1 testing, model time-horizon, the discounting of costs and outcomes and the impact of application of alternative plausible parametric functions to the extrapolation of OS and PFS. Subgroup analyses were also conducted to evaluate the cost-effectiveness of pembrolizumab in patient population with PD-L1 TPS $\geq 50 \%$ and TPS: $1-49 \%$.

\section{Results}

Base-case results

Base-case results (Table 4) projected greater LYs (0.60) and more QALYs (0.49) for patients receiving pembrolizumab compared with those receiving platinum-based chemotherapy. Pembrolizumab was also projected to be associated with higher overall costs, mainly driven by drug acquisition costs. The incremental cost per QALY gained with pembrolizumab over chemotherapy was US\$130,155, and the incremental cost per LY gained was US\$106,617.

\section{Subgroup analyses}

For the subgroup of patients with PD-L1 TPS $\geq 50 \%$, pembrolizumab was associated with a LY gain of 0.94 years and QALY gain of 0.77 compared with platinum-based chemotherapy, over the 20 -year time horizon, which resulted in ICERs of US $\$ 91,063 / \mathrm{LY}$ and US $\$ 111,781 /$ QALY. For the subgroup with PD-L1 TPS: 1-49\%, the incremental LYs and QALYs were 0.34 and 0.28, respectively. And the ICERs were US\$134,227/LY and US\$161,546/QALY.

\section{Scenario analyses}

In the scenario with OS adjusted for patients who switched from platinum-based chemotherapy to pembrolizumab in KEYNOTE-042, the number of LYs gained was 0.84 and the number of QALYs gained was 0.68 over the 20-year time horizon, which resulted in ICERs of US $\$ 116,451 /$ LY and US $\$ 142,681$ /QALY for pembrolizumab versus chemotherapy. Using PFS as a proxy for ToT resulted in an ICER of US\$53,684/LY and US\$65,536/QALY. With a 5-year time horizon, the ICER was US\$192,556/QALY and with a 10-year time horizon the ICER was US $\$ 140,786 /$ QALY. Changes in the discount rate had little effect on the ICER. With a discount rate for costs of $0 \%$ 
Table 3. Sensitivity analysis parameters.

\begin{tabular}{|c|c|c|c|}
\hline Model parameter & Base-case value or source & DSA range & PSA distribution model \\
\hline PFS - pembrolizumab & 9 week KM + Weibull onwards & $\begin{array}{l}95 \% \mathrm{Cl} \text { limits of the parameter } \\
\text { estimates in the Weibull function }\end{array}$ & $\begin{array}{l}\text { Random numbers generated from } \\
\text { multivariate normal distribution model }\end{array}$ \\
\hline PFS - chemotherapy & 9 week KM + exponential onwards & $\begin{array}{l}95 \% \mathrm{Cl} \text { limits of the parameter } \\
\text { estimates in the exponential function }\end{array}$ & $\begin{array}{l}\text { Random numbers generated from } \\
\text { multivariate normal distribution model }\end{array}$ \\
\hline ToT - pembrolizumab & KM curve & $95 \% \mathrm{Cl}$ limits of the $\mathrm{KM}$ curve & $\begin{array}{l}\text { Random numbers generated from the } \\
\text { upper and lower bounds of the KM curve }\end{array}$ \\
\hline ToT - chemotherapy & KM curve & $95 \% \mathrm{Cl}$ limits of the KM curve & $\begin{array}{l}\text { Random numbers generated from the } \\
\text { upper and lower bounds of the KM curve }\end{array}$ \\
\hline OS - chemotherapy & 33 week KM + exponential model & $\begin{array}{l}95 \% \mathrm{Cl} \text { limits of the parameter estimate } \\
\text { in the exponential function }\end{array}$ & $\begin{array}{l}\text { Random numbers generated from } \\
\text { multivariate normal distribution model }\end{array}$ \\
\hline Utilities & Based on KN024 trial data & $\pm 20 \%$ & $\begin{array}{l}\text { Beta distributions using the mean and SE } \\
\text { estimated from the KN024 trial }\end{array}$ \\
\hline Disease management cost in PD & Based on SEER Medicare & $\pm 25 \%$ & $\begin{array}{l}\text { Lognormal distribution with the SE set at } \\
20 \% \text { of the base-case value }\end{array}$ \\
\hline $\begin{array}{l}\text { Cost of subsequent active therapies - } \\
\text { pembrolizumab }\end{array}$ & US $\$ 9940$ & $\pm 25 \%$ & $\begin{array}{l}\text { Lognormal distribution with the SE set at } \\
20 \% \text { of the base-case value }\end{array}$ \\
\hline $\begin{array}{l}\text { Cost of subsequent active therapies - } \\
\text { chemotherapy }\end{array}$ & US $\$ 23,871$ & $\pm 25 \%$ & $\begin{array}{l}\text { Lognormal distribution with the SE set at } \\
20 \% \text { of the base-case value }\end{array}$ \\
\hline Death-related cost & Based on SEER Medicare & $\pm 25 \%$ & $\begin{array}{l}\text { Lognormal distribution with the SE set at } \\
20 \% \text { of the base-case value }\end{array}$ \\
\hline AE management cost - pembrolizumab & US $\$ 987$ & $\pm 50 \%$ & $\begin{array}{l}\text { Lognormal distribution with the SE set at } \\
20 \% \text { of the base-case value }\end{array}$ \\
\hline AE management cost - chemotherapy & US $\$ 1403$ & $\pm 50 \%$ & $\begin{array}{l}\text { Lognormal distribution with the SE set at } \\
20 \% \text { of the base-case value }\end{array}$ \\
\hline
\end{tabular}

AE: Adverse event; DSA: Deterministic sensitivity analysis; KM: Kaplan-Meier; KNO24: KEYNOTE-024; OS: Overall survival; PD: Progressive disease; PF: Progression free; PFS: Progression-free survival; PSA: Probabilistic sensitivity analysis; SE: Standard error; SEER: Surveillance, Epidemiology and End Results; ToT: Time-on-treatment.

\begin{tabular}{|c|c|c|c|}
\hline & Chemotherapy & Pembrolizumab & $\begin{array}{l}\text { Incremental pembrolizumab vs } \\
\text { chemotherapy }\end{array}$ \\
\hline Life years & 1.73 & 2.33 & 0.60 \\
\hline $\begin{array}{l}\text { Expected time in progression free state } \\
\text { (months) }\end{array}$ & 10.02 & 12.43 & 2.41 \\
\hline $\begin{array}{l}\text { Expected time in progressive state } \\
\text { (months) }\end{array}$ & 10.74 & 15.52 & 4.78 \\
\hline Quality-adjusted life-years & 1.28 & 1.77 & 0.49 \\
\hline Costs & US $\$ 167,046$ & US $\$ 230,954$ & US $\$ 63,909$ \\
\hline Drug acquisition cost & US $\$ 34,140$ & US $\$ 96,927$ & US $\$ 62,787$ \\
\hline Pre-medication cost & US $\$ 1380$ & US $\$ 0$ & - US $\$ 1380$ \\
\hline Drug administration cost & US $\$ 1273$ & US $\$ 1491$ & US $\$ 218$ \\
\hline Disease management cost & US $\$ 89,156$ & US $\$ 106,108$ & US $\$ 16,952$ \\
\hline Postdiscontinuation therapy cost & US $\$ 23,759$ & US $\$ 9842$ & - US $\$ 13,917$ \\
\hline Terminal care cost & US $\$ 15,936$ & US $\$ 15,598$ & -US\$337 \\
\hline Adverse event cost & US $\$ 1403$ & US $\$ 987$ & - US $\$ 416$ \\
\hline \multicolumn{4}{|l|}{ Incremental cost-effectiveness ratio } \\
\hline Cost per life-year gained & & & US $\$ 106,617$ \\
\hline $\begin{array}{l}\text { Cost per quality-adjusted life-year } \\
\text { gained }\end{array}$ & & & US $\$ 130,155$ \\
\hline
\end{tabular}


Table 5. Effect of different parametric functions to extrapolate overall survival and progression-free survival.

\begin{tabular}{|lll|}
\hline Parameter extrapolated & Alternative distribution models & ICER (US $\$ /$ QALY) \\
\hline PFS pembrolizumab & 9-week KM + Generalized Gamma & US $\$ 129,570$ \\
\hline PFS chemotherapy & 9-week KM + Weibull & US $\$ 130,145$ \\
\hline OS pembrolizumab & Log-normal & US $\$ 104,033$ \\
\hline OS chemotherapy & Log-logistic & US $\$ 140,472$ \\
\hline Base case & & US $\$ 130,155$ \\
\hline
\end{tabular}

ICER: Incremental cost-effectiveness ratio; KM: Kaplan-Meier; OS: Overall survival; PFS: Progression-free survival; QALY: Quality-adjusted life-year.

and for outcomes of $5 \%$ (the least favorable combination) the ICER was US $\$ 150,953 /$ QALY, and with a discount rate for costs of $5 \%$ and for outcomes of $0 \%$ (the most favorable combination) the ICER was US $\$ 108,502 /$ QALY.

To be treated with pembrolizumab monotherapy, patients must have a PD-L1 test conducted, with the result demonstrating PD-L1 expression (TPS $\geq 1 \%$ ). The focus of these analyses has been to evaluate the cost-effectiveness of pembrolizumab for such patients who have been identified for treatment. Further examination of the policy of testing all patients for PD-L1, treating PD-L1 positive (TPS $\geq 1 \%$ ) patients with pembrolizumab and PD-L1 negative patients with chemotherapy, versus a no-testing strategy with all patients treated with chemotherapy was performed. In this scenario, pembrolizumab would be expected to result in an ICER of US $\$ 130,558 /$ QALY. Thus, including costs of PD-L1 testing has a very small impact on the model results.

Applying alternative plausible parametric functions to the extrapolation of OS and PFS from the trial data to the 20-year model time horizon did not have large effects on the results (Table 5). Using different cut-off points (week 28, 33, 42 and 52) in the two-phase piecewise exponential model for the OS extrapolation also yielded similar results, where the ICER ranged from US $\$ 135,912$ to US $\$ 128,796 /$ QALY.

\section{One-way \& probabilistic sensitivity analyses}

The tornado diagram depicted in Figure 5 below shows the impact of parameter variation on the cost/QALY ICER as derived from the one-way deterministic sensitivity analyses. The variables that had the most impact on the ICER were extrapolation of OS, utilities for time greater or equal to 360 days from death and disease management cost during first year after treatment initiation.

The PSA resulted in mean expected incremental costs of US\$64,066, mean expected incremental QALYs of 0.49 and a mean expected ICER of US $\$ 130,686 /$ QALY for pembrolizumab compared with platinum-based chemotherapy. Figure 6A shows the results of each iteration within a scatter plot, and Figure $6 \mathrm{~B}$ shows the cost-effectiveness acceptability curve. The probability the ICER would be below US $\$ 150,000 /$ QALY and US $\$ 190,000 /$ QALY was 69 and $90 \%$.

\section{Discussion}

The cost-effectiveness of pembrolizumab versus platinum-based chemotherapy has already been demonstrated for previously untreated metastatic NSCLC patients with a TPS $\geq 50 \%$ based on data from KEYNOTE-024 [13]. The reported ICER was US $\$$ US97,621/QALY or US\$US78,344/LY gained. This analysis evaluated the economic impact of pembrolizumab as first-line treatment for the extended advanced NSCLC population with TPS $\geq 1 \%$. Base-case results of the analysis indicated that compared with platinum-based chemotherapy over a 20-year time horizon, pembrolizumab was expected to result in an additional 0.60 LYs and an additional 0.49 QALYs, at an additional cost of US\$73,756, giving an incremental cost of US\$130,155/QALY gained or an incremental cost of US\$106,617/LY gained. The main driver of the increased cost with pembrolizumab was the drug acquisition cost. The ICER was most sensitive to the extrapolation of OS, utility values and cost in the progression-free state.

There has been no general agreement on a cost-effectiveness ratio threshold for the US. Braithwaite et al. [34] cite multiple decision rules, including the commonly cited US\$50,000/QALY gained and the WHO criteria of three-times the gross domestic product (GDP) per capita per disability-adjusted LY gained [35], which is around US $\$ 194,000$ in 2019 [36]. A more recent analysis by Neumann and colleagues recommended using a QALY range of between US $\$ 100,000$ and US $\$ 150,000 /$ QALY gained, although analyses should allow for examination of multiple thresholds up to US\$200,000/QALY gained [37]. The base-case ICER for pembrolizumab of US\$130,155/QALY in the current analysis falls within these ranges of acceptable thresholds. Results from the PSA showed a $69 \%$ probability that the ICER would be below US $\$ 150,000 /$ QALY and a 90\% probability of being below US $\$ 190,000 /$ QALY. 


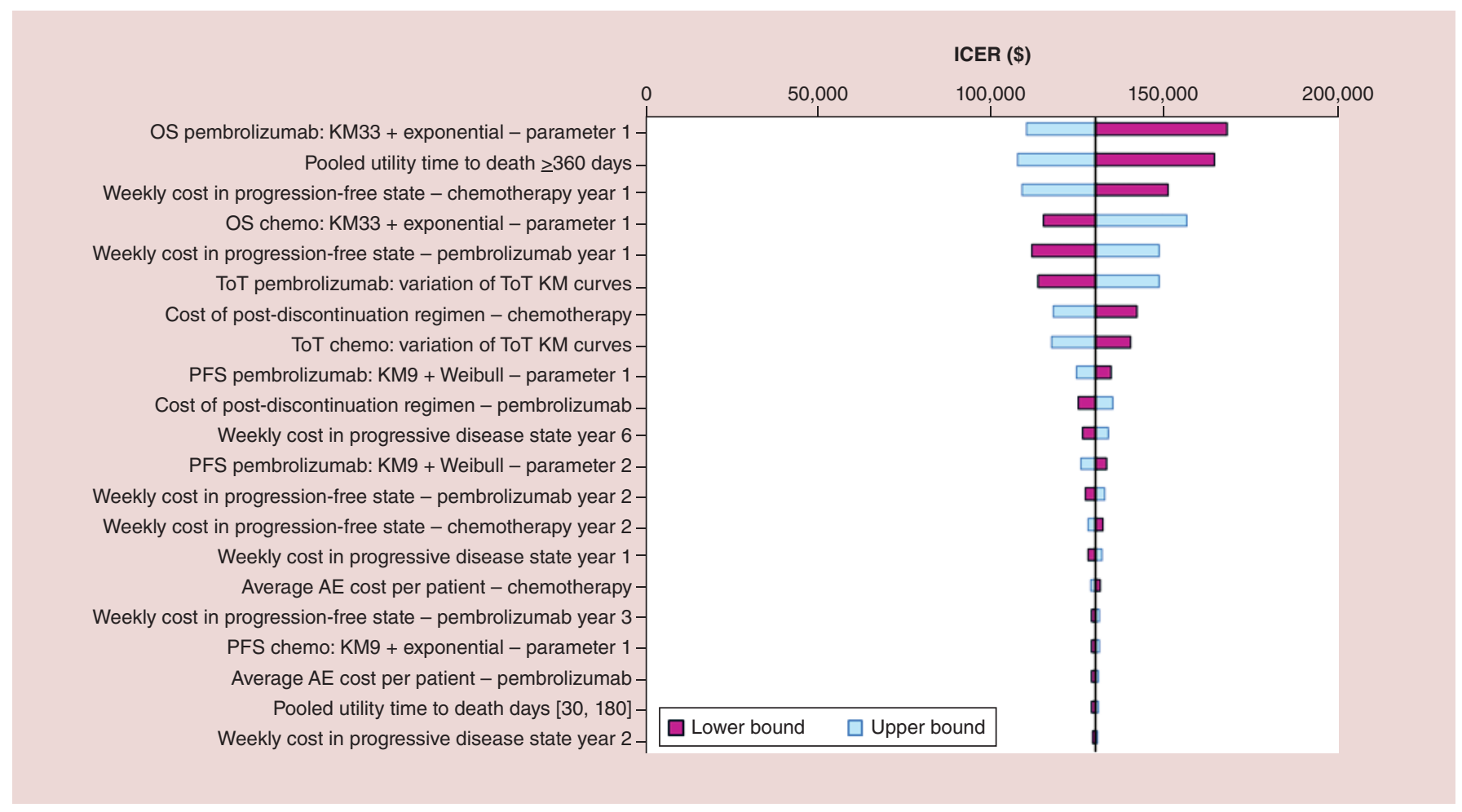

Figure 5. One-way sensitivity analysis tornado diagram for the incremental cost-effectiveness ratio (per quality-adjusted life-year) of pembrolizumab versus platinum-based chemotherapy.

chemo: Platinum-based chemotherapy; ICER: Incremental cost-effectiveness ratio; KM: Kaplan-Meier; OS: Overall survival; Parameter 1: Intercept parameter derived by R program for exponential/Weibull functions; PFS: Progression-free survival; QALY: Quality-adjusted life-year; ToT: Time-on-treatment; Weekly cost: Weekly disease management cost.

The subgroup analyses suggested that the ICER was US $\$ 111,781 /$ QALY for pembrolizumab compared with platinum-based chemotherapy in patients with TPS $\geq 50 \%$, which was consistent with the results (ICER of US $\$ 97,621$ /QALY) derived from the previous cost-effectiveness analysis based on KEYNOTE-024 for a similar population [13]. The analyses showed a more favourable ICER for patients with TPS $\geq 50 \%$ compared with those with TPS: $1-49 \%$. This is primarily driven by greater magnitude of survival benefit for pembrolizumab at higher levels of PD-L1 expression. However, based on the threshold of three-times the GDP per capita (US $\$ 194,000 /$ QALY), pembrolizumab was projected to be cost-effective (ICER: US $\$ 111,781 /$ QALY and US $\$ 161,546 /$ QALY) for both subgroups.

Pembrolizumab has also been approved in the US for use in combination with chemotherapy for patients, regardless of PD-L1 status, and the availability of data from KEYNOTE-042 may generate questions regarding choice of pembrolizumab monotherapy versus pembrolizumab and chemotherapy combinations. Though not the subject of the present evaluation, an ICER of US\$56,112/QALY was reported for pembrolizumab + chemotherapy versus pembrolizumab monotherapy in US patients with metastatic squamous NSCLC and PD-L1 expression of $1-49 \%$ [38], incorporating data from KEYNOTE-042 for monotherapy. For squamous patients with PD-L1 $\geq 50 \%$, though a nominally cost saving result was reported for pembrolizumab + chemotherapy versus pembrolizumab monotherapy, the difference in incremental QALYs was close to zero, suggesting a need for further evidence to better define cost-effectiveness between these therapeutic options. For nonsquamous metastatic NSCLC patients, in patients with PD-L1 $\geq 50 \%$, an ICER of US $\$ 147,365 /$ QALY was previously reported for pembrolizumab + chemotherapy versus pembrolizumab monotherapy [39]. Using the same model, an ICER of US $\$ 122,220 /$ QALY has been observed in the PD-L1 1-49\% sub-group. Based on these results, either pembrolizumab + chemotherapy or pembrolizumab monotherapy may currently be considered a reasonable, costeffective option for patients in these populations, depending on decision-makers' cost-effectiveness threshold and physician and patient preferences for therapy. 


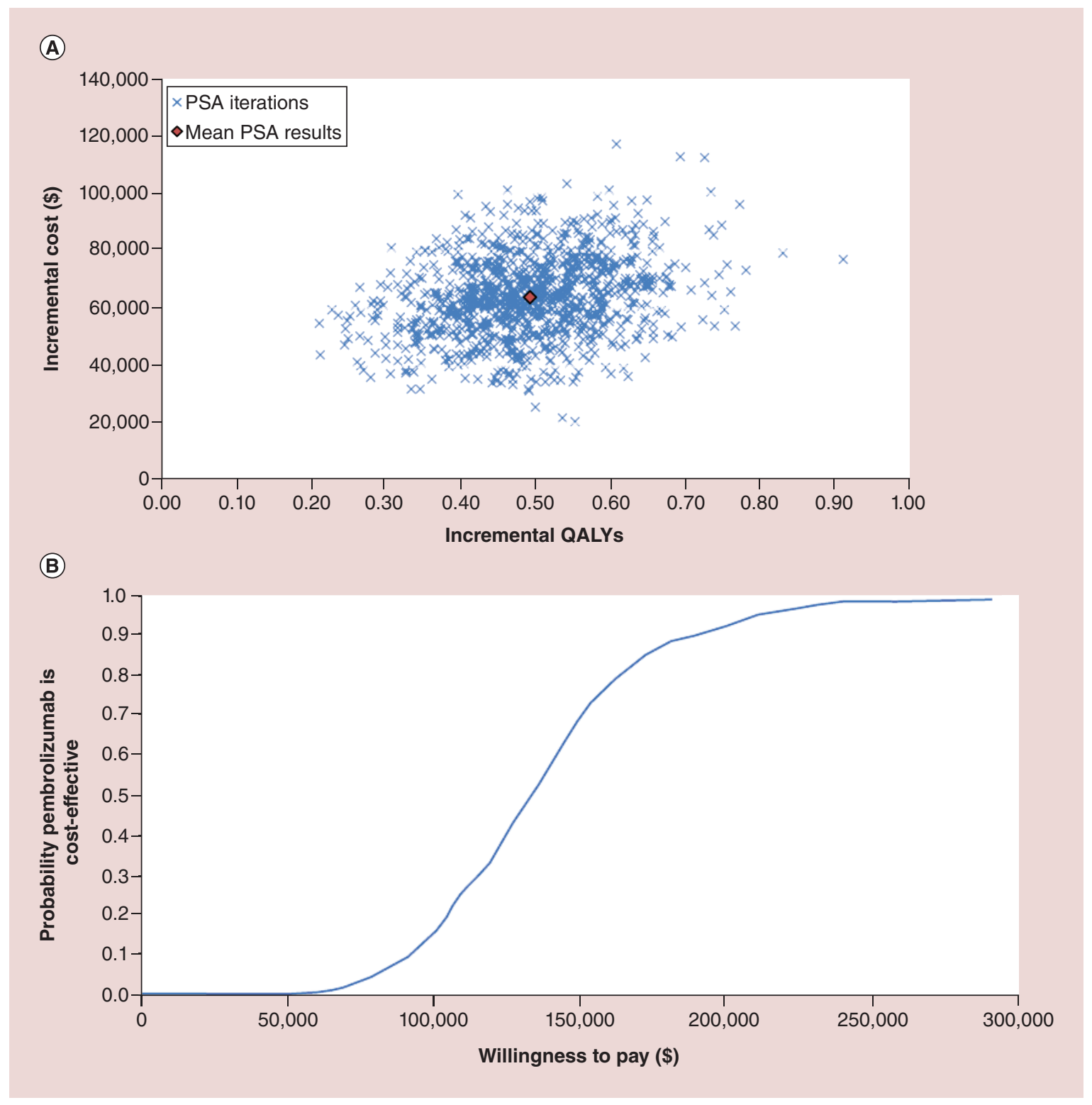

Figure 6. Probabilistic sensitivity analysis. (A) Cost-effectiveness plane. (B) Cost-effectiveness acceptability curve. PSA: Probabilistic sensitivity analysis; QALY: Quality-adjusted life-year.

The base-case analysis was based on the ITT population, which included patients who switched from chemotherapy to pembrolizumab and other PD1/PD-L1 inhibitors after progression. The effect of switching diluted the survival benefit associated with pembrolizumab, while increased costs in the chemotherapy group. A scenario analysis was conducted using switching adjusted OS data and resulted in higher estimates for LYs and QALYs gained and a higher ICER (US\$142,681/QALY), compared with the base case (US\$130,155/QALY).

This analysis was based on the efficacy and safety data from a randomized clinical trial directly comparing pembrolizumab and chemotherapy, which is considered as a key strength. The analysis also has several limitations. The KEYNOTE-042 trial, which was conducted outside the US, may not reflect US clinical practice. In the KEYNOTE-0 2 trial, $52.3 \%$ of nonsquamous patients in the chemotherapy arm received pemetrexed maintenance therapy. This compares to $26.6 \%$ reported in the US Flatiron database for nonsquamous patients receiving chemotherapy induction therapy who received maintenance therapy [30]. As the impact on efficacy associated with these different rates is not known, the impact on the ICER results is also unknown. KEYNOTE-042 was conducted mostly in Asia-Pacific, Eastern Europe and South America, with 29\% of patients enrolled in east Asia. 
These are regions where there was less availability of and access to therapy, particularly immunotherapy [11]. In KEYNOTE-042, 20.7\% of patients in the chemotherapy arm received PD-1/PD-L1 inhibitors as subsequent therapy. Estimations from the Flatiron database [40] (in patients diagnosed between 1 February 2015 and 31 December 2017) showed that $58.6 \%$ of advanced NSCLC patients with PD-L1 $\geq 1 \%$ who received platinumbased chemotherapy as first-line therapy subsequently received PD-1/PD-L1 inhibitors. Higher switching rate is expected to result in longer survival and higher cost for these patients, and the bias to the ICER results could be in either direction. However, the analysis reported higher ICERs when treatment switching was adjusted, which suggested that less switching in the chemotherapy arm led to higher ICERs for pembrolizumab. Therefore, the higher rate of subsequent PD-1/PD-L1 regimen use in Flatiron database compared with the switching data from KEYNOTE-042 is projected to result in lower ICERs for pembrolizumab than in the model base case results.

The cost of grade 3-5 AEs with a risk of lower than 5\% in both treatment groups was not included in the analysis. This leads to underestimation of AE costs. However, the bias is not expected to be large considering the low risk of the excluded AEs. AE related drug costs were not considered in the model either, as the main driver of the AE management cost is hospitalization. Sensitivity analyses have been conducted to vary the AE cost by $\pm 50 \%$ and the results show that the variation of AE costs has a small impact on the ICER.

At the data cut-off date from KEYNOTE-042 for this analysis, the median follow-up in the trial was about 14 months. Extrapolation was necessary to model a lifetime time horizon and uncertainty is inherent in this process. Although several survival curve options were explored in scenario analyses, longer-term data will be important to better understand long-term outcomes.

\section{Conclusion}

Compared with platinum-based chemotherapy, pembrolizumab demonstrated superior OS and improved tolerability in previously untreated advanced NSCLC patients whose tumors express PD-L1 (TPS $\geq 1 \%$ ). ICERs were well below a three-times US GDP per capita threshold within the full trial population as well as PD-L1 subgroups of $\geq 50 \%$ and $1-49 \%$. Pembrolizumab monotherapy is projected to improve life expectancy and expected qualityadjusted life time. Based on the WHO threshold of three-times GDP per capita, pembrolizumab can be considered as a cost-effective option compared with platinum-based chemotherapy in these biomarker-identified advanced NSCLC patients from a third-party US public payer perspective.

\section{Summary points}

- Previous studies have demonstrated that first-line pembrolizumab monotherapy significantly improves overall survival and is projected to be cost effective compared with platinum-based chemotherapy in patients with untreated metastatic non-small-cell lung cancer (NSCLC) with a programmed death ligand 1 (PD-L1) tumor proportion score $\geq 50 \%$ from a US payer perspective.

- This study evaluated the cost-effectiveness of pembrolizumab monotherapy compared with platinum-based chemotherapy when extended as first-line treatment for advanced NSCLC with PD-L1 tumor proportion score $\geq 1 \%$.

- A partitioned-survival model was developed using data from the KEYNOTE-042 randomized clinical trial. Kaplan-Meier estimates of time-on-treatment, progression-free survival and overall survival were used in the model with extrapolation based on parametric models and validation with long-term registry data.

- Costs (US $\$ 2019$ value) for drug acquisition/administration, adverse events and clinical management were included in the model. Health outcomes were measured by expected life years and quality-adjusted life-years (QALYs). Costs and health outcomes were discounted at 3\% per year.

- In the base-case scenario, pembrolizumab resulted in an expected gain of 0.60 life-years (LYs) and 0.49 QALYs, and an incremental cost of US $\$ 63,925$ compared with platinum-based chemotherapy.

- The incremental cost per QALY gained was US $\$ 130,155 /$ QALY and the incremental cost per LY gained was US $\$ 106,617 /$ LY, which is lower than the cost-effectiveness threshold of three-times 2019 gross domestic product per capita for the US (US\$194,000).

- Probabilistic sensitivity analyses showed over $90 \%$ chance that the incremental cost-effectiveness ratios would be below this threshold.

- Pembrolizumab increased expected quality-adjusted life-years in patients with PD-L1-positive advanced NSCLC and is projected to be cost effective from a US third-party public healthcare payer perspective compared with platinum-based chemotherapy at published thresholds of cost-effectiveness. 


\section{Author contributions}

M Huang led the development of this health economic analysis and initiated the manuscript draft. All authors contributed to the study design and methodology, interpretation of the results and development and writing of the draft manuscript and approved the final version.

\section{Acknowledgments}

The authors thank A Chakravarty of Complete HEOR Solutions (CHEORS), who provided data analytics support for this study.

\section{Financial \& competing interests disclosure}

This study and manuscript were funded by Merck Sharp \& Dohme Corp., a subsidiary of Merck \& Co. Inc., Kenilworth, NJ, USA. M Huang, R Insinga, T Burke, F Ejzykowicz and $Y$ Zhang are employees of Merck Sharp \& Dohme Corp., a subsidiary of Merck \& Co. Inc., Kenilworth, NJ, USA, the sponsor of this study and manuscript. G Lima Lopes provides advisory and consultancy services to Merck \& Co. Inc. J Feliciano provides advisory and consultancy services to Merck \& Co. Inc, EliLilly, AstraZeneca, Pfizer, and Takeda; and receives research funding from Bristol-Myers Squibb and AstraZeneca. The authors have no other relevant affiliations or financial involvement with any organization or entity with a financial interest in or financial conflict with the subject matter or materials discussed in the manuscript apart from those disclosed.

No writing assistance was utilized in the production of this manuscript.

\section{Open access}

This work is licensed under the Creative Commons Attribution-NonCommercial 4.0 Unported License. To view a copy of this license, visit http://creativecommons.org/licenses/by-nc-nd/4.0/

\section{References}

Papers of special note have been highlighted as: • of interest

1. Torre LA, Siegel RL, Ward EM, Jemal A. Global cancer incidence and mortality rates and trends-an update. Cancer Epidemiol. Biomarkers Prev. 25(1), 16-27 (2016).

2. World Health Organization (WHO). Non-Small-Cell Lung Cancer. www.who.int/selection_medicines/committees/expert/20/applications/NonSmallCellLungCancer.pdf

3. National Comprehensive Cancer Network (NCCN). NCCN clinical practice guidelines in oncology: non-small cell lung cancer. Version 5.2019. www.nccn.org/professionals/physician_gls/default.aspx

4. Cho JH. Immunotherapy for non-small-cell lung cancer: current status and future obstacles. Immune Netw. 17(6), 378-391 (2017).

5. Reck M, Rodríguez-Abreu D, Robinson AG et al. Pembrolizumab versus chemotherapy for PD-L1-positive non-small-cell lung cancer. N. Engl. J. Med. 375(19), 1823-1833 (2016).

6. Reck M, Rodriguez-Abreu D, Robinson AG et al. Updated analysis of KEYNOTE-024: pembrolizumab versus platinum-based chemotherapy for advanced non-small-cell lung cancer with PD-L1 tumor proportion score of 50\% or greater. J. Clin. Oncol. 37(7), 537-546 (2019).

7. Herbst RS, Baas P, Kim DW et al. Pembrolizumab versus docetaxel for previously treated, PD-L1-positive, advanced non-small-cell lung cancer (KEYNOTE 010): a randomised controlled trial. Lancet 387(10027), 1540-1550 (2016).

8. Gandhi L, Rodríguez-Abreu D, Gadgeel S et al. Pembrolizumab plus chemotherapy in metastatic non-small-cell lung cancer. N. Engl. J. Med. 378(22), 2078-2092 (2018).

9. Paz-Ares L, Luft A, Vicente D et al. Pembrolizumab plus chemotherapy for squamous non-small-cell lung cancer. N. Engl. J. Med. 379(21), 2040-2051 (2018).

10. FDA KEYTRUDA Prescribing Information (2019). www.accessdata.fda.gov/drugsatfda_docs/label/2019/125514s040lbl.pdf

- Reference for indicated population/dosing schedule of pembrolizumab by US FDA.

11. Mok T, Wu Y, Kudaba I et al. Pembrolizumab versus chemotherapy for previously untreated, PD-L1-expressing, locally advanced or metastatic non-small-cell lung cancer (KEYNOTE-042): a randomised, open-label, controlled, phase 3 trial. Lancet 393(10183), 1819-1830 (2019).

\section{- Primary clinical publication of the KEYNOTE-042 trial.}

12. Mok TSK, Wu Y-L, Kudaba I et al. Final analysis of the phase III KEYNOTE-042 study: pembrolizumab (Pembro) versus platinum-based chemotherapy (Chemo) as first-line therapy for patients (Pts) with PD-L1-positive locally advanced/metastatic NSCLC. Ann. Oncol. 30(Suppl. 2), (2019).

- Clinical publication of final analysis of KEYNOTE-042, which is the main data source of this analysis.

13. Huang M, Lou Y, Pellissier J et al. Cost effectiveness of pembrolizumab vs standard-of-care chemotherapy as first-line treatment for metastatic NSCLC that expresses high levels of PD-L1 in the United States. Pharmacoeconomics 35(8), 831-844 (2017). 
14. Partitioned Survival Model [online]. York Health Economics Consortium (2016). www.yhec.co.uk/glossary/partitioned-survival-model/

15. National Cancer Institute. Common Terminology Criteria for Adverse Events (CTCAE) Version 4.0. www.eortc.be/services/doc/ctcl

16. Sanders GD, Neumann PJ, Basu A et al. Recommendations for conduct, methodological practices, and reporting of cost-effectiveness analyses: second panel on cost-effectiveness in health and medicine. JAMA 316(10), 1093-1103 (2016)

17. Eisenhauer EA, Therasse P, Bogaerts J et al. New response evaluation criteria in solid tumors: revised RECIST guideline (version 1.1). Eur. J. Cancer 45, 228-247 (2009).

18. Latimer NR. NICE DSU Technical Support Document 14: survival analysis for economic evaluations alongside clinical trials extrapolation with patient-level data (2011).

- Guideline for survival extrapolation, based on which the parametric model fitting was carried out.

19. Latimer NR, Abrams KR. NICE DSU Technical Support Document 16: adjusting survival time estimates in the presence of treatment switching. http://nicedsu.org.uk/wp-content/uploads/2016/03/TSD16_Treatment_Switching.pdf

20. Latimer NR. Survival analysis for economic evaluations alongside clinical trials - extrapolation with patient-level data: inconsistencies, limitations, and a practical guide. Med. Decis. Making 33(6), 743-754 (2013).

21. Chow GC. Tests of equality between sets of coefficients in two linear regressions. Econometrica 28(3), 15 (1960).

22. National Cancer Institute. National Cancer Institute SEER Data, 1973-2015. http://seer.cancer.gov/data

23. Arunachalam A, Li H, Bittoni MA et al. Real-world treatment patterns, overall survival, and occurrence and costs of adverse events associated with second-line therapies for Medicare patients with advanced non-small-cell lung cancer. Clin. Lung Cancer 19(5), e783-e799 (2018).

24. Bittoni MA, Arunachalam A, Li H et al. Real-world treatment patterns, overall survival, and occurrence and costs of adverse events associated with first-line therapies for Medicare patients 65 years and older with advanced non-small-cell lung cancer: a retrospective study. Clin. Lung Cancer 19(5), e629-e645 (2018).

25. Huang M, Chandwani S, Insinga R et al. PCN345-Health State Utilities in Metastatic NSCLC: a study of multiple immuno-oncology trials. Value Health 21, S72-S73 (2018).

- Reference for health state utility values used in this analysis.

26. Hatswell AJ, Pennington B, Pericleous L, Rowen D, Lebmeier M, Lee D. Patient-reported utilities in advanced or metastatic melanoma, including analysis of utilities by time to death. Health Qual. Life Outcomes 12, 140 (2014).

27. Center for Medicare and Medicaid Services. Medicare 2017 costs at a glance(2017). www.medicare.gov/your-medicare-costs/costs-at-a-glance/costs-at-glance.html\#collapse-4809

28. Bureau of Labor Statistics Consumer Price Index - All Urban Consumers (2019). www.bls.gov/cpi/

29. Analysource. www.Analysource.com

30. Abernethy AP, Arunachalam A, Burke T et al. Real-world first-line treatment and overall survival in non-small cell lung cancer without known EGFR mutations or ALK rearrangements in US community oncology setting. PloS ONE 12(6), e0178420 (2017).

31. NCCN Clinical Practice Guidelines in Oncology: Version

1.2017. www.vitromolecularlaboratories.com/wp-content/uploads/2016/10/NCCN-1.2017-NSCLC-Guidelines.pdf

32. Center for Medicare and Medicaid Services

2019. www.cms.gov/Medicare/Medicare-Fee-for-Service-Payment/AcuteInpatientPPS/FY2019-IPPS-Final-Rule-Home-Page.html

33. Center for Medicare and Medicaid Services. Costs for Hospital Outpatient Services, by HCPCS code for CY (2019). www.cms.gov/Medi care/Medicare-Fee-for-Service-Payment/HospitalOutpatientPPS/Downloads/CMS-1695-FC-2019-OPPS-FR-Claims-Accounting.pdf

34. Braithwaite RS, Meltzer DO, King JT Jr, Leslie D, Roberts MS. What does the value of modern medicine say about the $\$ 50,000$ per quality-adjusted life-year decision rule? Med. Care 46(4), 349-356 (2008).

35. World Health Organization (WHO). Choosing interventions that are cost-effective. www.who.int/choice/en/

- Reference for the cost-effectiveness threshold used for this analysis.

36. KNOEMA. IMF World Economic Outlook (WEO). https://knoema.com/pjeqzh/gdp-per-capita-by-country-1980-2014

37. Neumann PJ, Cohen JT, Weinstein MC. Updating cost-effectiveness - the curious resilience of the $\$ 50,000$-per-QALY threshold. $N$. Engl. J. Med. 371(9), 796-797 (2014).

- Another important reference for the cost-effectiveness thresholds in the USA.

38. Insinga RP, Vanness DJ, Feliciano JL et al. Cost-effectiveness of pembrolizumab in combination with chemotherapy versus chemotherapy and pembrolizumab monotherapy in the 1st-line treatment of squamous NSCLC in the US. Curr. Med. Res. Opin. 35(7), $1241-1256$ (2019).

39. Insinga RP, Vanness DJ, Feliciano JL et al. Cost-effectiveness of pembrolizumab in combination with chemotherapy in the 1st line treatment of non-squamous NSCLC in the US. J. Med. Econ. 21(12), 1191-1205 (2018).

40. Flatiron Health. https://flatiron.com/real-world-evidence/ 